\title{
Design of rapid monitoring system of geological disaster based on LoRa
}

\author{
Wang Chenhui ${ }^{1,}{ }^{*}$ and Meng Qingjia ${ }^{1}$ \\ ${ }^{1}$ Center for Hydrogeology and Environmental Geology Survey, China Geological Survey, Baoding, Hebei, China
}

\begin{abstract}
According to the characteristics of the geological environment and disasters, this paper uses microelectronics, wireless communication, thin-film solar power supply and other technologies, combined with lightweight process design, a new scheme for rapid geological disaster monitoring system based on LoRa is proposed. The system is based on embedded microprocessor of STM32F103 and SX1278 module of LoRa, which uses self-organizing network design of star-type and constructs a monitoring system with long communication distance and stable and reliable data transmission. The system can realize real-time data collection of multiple monitoring parameters of the disaster body and transmit the monitoring data to the data center or dedicated data receiving terminal through LoRa/GPRS/BeiDou satellite, which can provide data support for expert analysis and decision-making. The system has the features of low power consumption, long transmission distance, ad hoc network, stable and reliable communication, which has wide application prospect in the field of geological disaster monitoring.
\end{abstract}

\section{Introduction}

Geological disaster is natural disaster caused by geological dynamic activities or abnormal changes in geological environment. Geological disasters will cause huge losses to human life and property. The most common geological disasters include collapse, landslide and debris flow, etc. The monitoring methods for the above types of geological disasters mainly include monitoring methods such as rainfall monitoring, surface crack displacement monitoring, and water content monitoring. At present, there is a lack of means for rapid monitoring of geological disasters, especially the rapid monitoring systems for geological disasters that can be deployed on-site before or after disasters.

At this stage, the conventional deployment method of geological disaster monitoring is to install and deploy relevant geological disaster monitoring and early warning instruments at the hidden points of geological disasters. Geological disaster monitoring and early warning instruments are mainly composed of data acquisition system, transmission system, power supply system and protection system.

Currently, the shortcomings of this type of conventional geological disaster monitoring and early warning instrument are that the instrument has large weight, large volume, high installation and transportation cost, long time to obtain disaster body monitoring data, and can't be replaced in a short time, that has brought many inconveniences to geological disaster monitoring. Therefore, it is urgent to develop a geological disaster monitoring system that can be easily carried, quickly deployed, and quickly acquire data.
The development of the geological disaster rapid monitoring system and the application of related technologies can meet the monitoring requirements of hidden geological hazards and critical geological disasters. Its design features of light and easy to carry, easy to use, fast deployment, complete functions, and reliable performance are accordance with the requirements of rapid disaster monitoring.

Once the geological disaster occurred, monitoring personnel can carry the rapid monitoring system to enter the site according to the results of the rapid survey. Furthermore, the equipment was installed and evacuated, that the collected data was transmitted to the monitoring center in real-time through the data link of the BeiDou and GPRS communications, which can be provided to the leaders and experts for decision analysis. The rapid geological disaster monitoring system can simultaneously collect monitoring parameters such as disaster point rainfall, surface crack displacement, and video monitoring.

For the sudden geological disasters, rapid monitoring sensors can be realized rapid deployment and rapid monitoring, which need to be improved so that the sensor functional structures can be flexibly layout during the disaster phase and the corresponding monitoring parameters can be quickly achieved. The monitoring data can truly and effectively reflect the monitoring factors such as rainfall, surface deformation, and status change of the source area.

At present there are several problems in the rapid monitoring: (1) Insufficient signal coverage, many devices are deployed in areas with sparse population or complex environments. The operator network covers 
blind spots or lacks signal strength, which making it difficult to ensure stable data transmission. (2) High power consumption, a large number of equipment require battery power. If a cellular network is used and frequent battery replacement is required, which is difficult to implement in many harsh environments. (3) Low cost effectiveness, the device transmits a small amount of data and transmission frequency is very low, at present, cellular networks are designed for high bandwidth. The use of cellular networks takes up network and code number resources, and it also generates monthly traffic fees.

The following key problems are need to be solved in this paper: (1) The lightweight design of the rapid geological disaster monitoring system, which can be achieved to improve the rapid monitoring system and process of monitoring the sensor shell, which is more in line with the characteristics of emergency monitoring, achieving a compact size and complete functions. (2) Wireless sensor design, it is need to eliminate the wired connection scheme of the existing sensors and the intelligent monitoring terminal for geological disaster fast monitoring, furthermore, it is need to realize the wireless connection between the terminal and sensor, and realize fast access monitoring data. (3) Appearance of the sensor process design, according to the characteristics of the monitoring elements, the structure of the sensor is improved to realize rapid development.

\section{Methods and Approach}

The system mainly includes front-end monitoring sensor, RF transmission modules of LoRa, network nodes, GPRS networks, geological disaster monitoring and warning cloud severs[1]. The network nodes include onsite acquisition nodes, relay nodes (if necessary) and aggregation nodes. The on-site acquisition nodes are responsible for the collection of geological disasters sensor data (such as rainfall, displacement, and tilt sensor). The aggregation node is mainly responsible for sending and receiving data of the acquisition node and uploads the data to the geological disaster monitoring and warning cloud server through GPRS or BeiDou.

One side, acquisition nodes collects the on-site monitoring data accurately, stably, and reliably according to the selection of sensor types input, on the other side, acquisition nodes can communicate with the aggregation node by using long distance wireless communication of LoRa networks and transmit the data to the cloud server through the aggregation node.

The geological disaster monitoring and warning cloud server not only can make analysis of the on-site data, but also can intelligently judge the status of the connected sensors, and integrate the monitoring data through the established data algorithm to analyze the specific conditions and deformation trends of the geological disaster.

The system can realize rapid deployment and fast access to data. Furthermore, the system can be powered on and its network architecture is simple. It not only provides data query and management for experts, also provides intelligent decision-making basis and effectively improves the efficiency of monitoring and early warning of geological disasters[2-4]. The diagram of rapid monitoring system is shown in Figure 1.

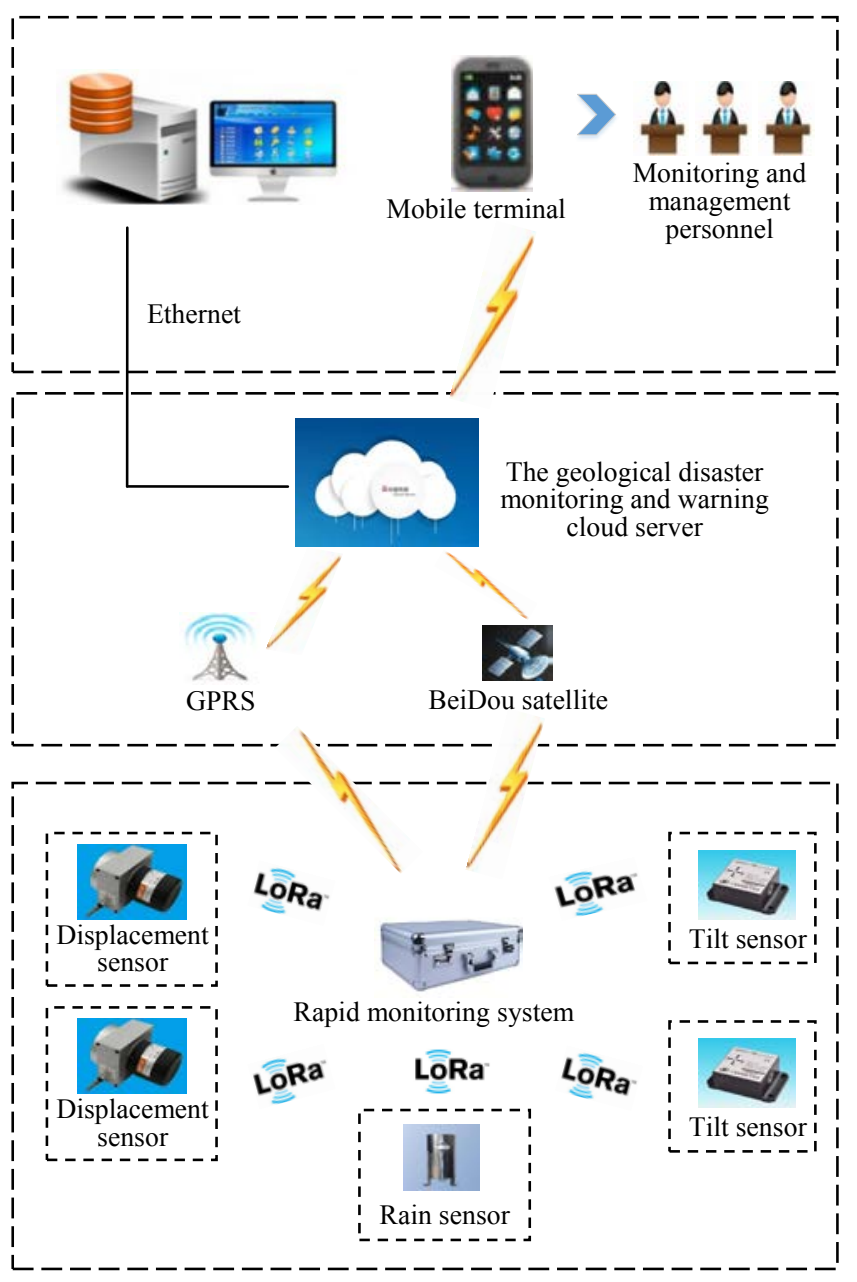

Fig. 1. The diagram of rapid monitoring system

\section{Hardware Design of Rapid Monitoring System}

\subsection{Microprocessor}

STM32F103 devices use the Cortex-M3 core, with a maximum CPU speed of $72 \mathrm{MHz}$. The portfolio covers from 16 Kbytes to 1 Mbyte of Flash with motor control peripherals, USB full-speed interface and CAN. It integrates a variety of high-performance industrial interconnect standard interfaces, which has 6-12 clock cycles and realizes fast nested interrupts. In addition, STM32F103 has MPU protection set access rules and perfect compatibility and can adapt to a variety of IOT applications.

\subsection{Selection of LoRa Module}

LoRa is a kind of LPWAN communication technology. It is an ultra-long-distance wireless transmission scheme based on spread spectrum technology, which is adopted 
and promoted by Semtech of the United States. It effectively solves the problem that the traditional wireless sensor network cannot have both the transmission distance and the low power consumption.

The main technical features of LoRa are as follows:

- Strong compatibility, all applications that meet the LoRa WAN protocol can access.

- Access is flexible, single gateway can access dozens to tens of thousands of nodes, nodes can randomly access the network and the number of nodes can be extended.

- Strong concurrency, the gateway can support at least 8 frequency points, while random 8-way data concurrency, frequency point can be extended.

- Full-duplex communication can be achieved, with no conflict between uplink and downlink, and strong practical effect.

- Network topology is simple, the star network has higher reliability and lower power consumption.

In this article we select KT-F1278 as communication module of LoRa. KT-F1278 wireless module adopts Semtech company SX1278 device, the device adopts the LoRa TM frequency-hopping spread spectrum modulation technology. Its communication distance and receiving sensitivity are far above the FSK, GFSK modulation, and its multiple transmission of signal occupy the same channel and the channel is unaffected. KT-F1278 can significantly extend the transmission distance. It can reach $15 \mathrm{~km}$ in sparse environment and 3 $\mathrm{km}$ in densely populated areas. Therefore, there is no need for relays and complicated communication infrastructure in this system.

\subsection{Design of the acquisition node}

According to the characteristics of the geological disaster rapid monitoring system, the acquisition node is mainly responsible for the data collection of each monitoring sensor at the geological disaster site[5-7]. The schematic diagram of the acquisition node structure is shown in Figure 2.

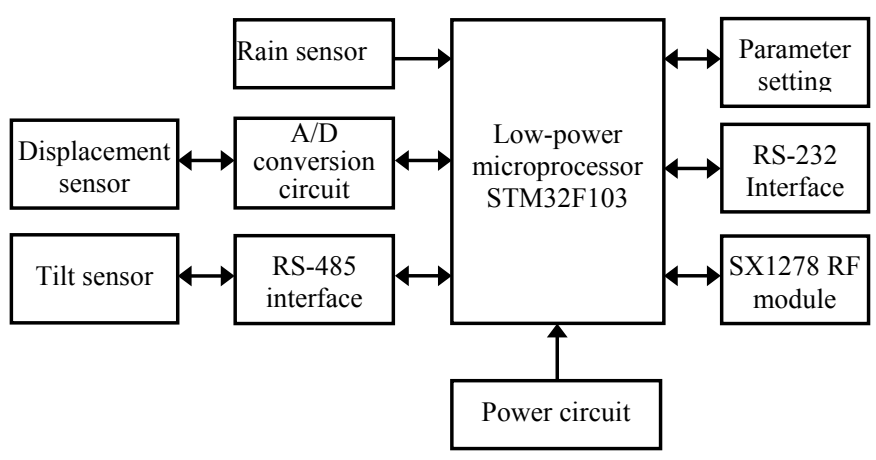

Fig. 2. The schematic diagram of the acquisition node structure On the one hand, the acquisition node is responsible for the collection and control of data such as on-site rainfall sensor, surface displacement sensor, and tile sensor. On the other hand, it needs to respond to the control instructions issued by the convergence nodes and upload the collected dat a to the convergence nodes to achieve real-time data acquisition, control and storage of geological disasters on the spot.

The rain sensor is connected to the IO port of microprocessor for data acquisition. The surface displacement sensor accesses the ADS1256 for data acquisition. The tilt sensor uses the RS-485 interface for data acquisition[8-9].

\subsection{Design of the convergence node}

The convergence node is responsible for the wireless network instruction issued, data reception and upload, detection of the system and management, and other functions.

The convergence node receives the field data uploaded by the acquisition nodes in the area through the LoRa wireless network, and uploads the data to the geological disaster monitoring and warning cloud server via the GPRS network or the BeiDou satellite network; at the same time, it sends the acquisition and control commands to any collection node of the LoRa network. The schematic diagram of the convergence node structure is shown in Figure 3.

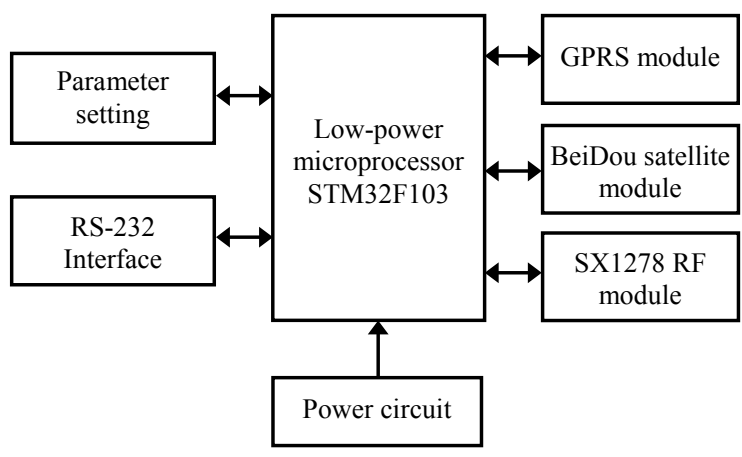

Fig. 3. The schematic diagram of the convergence node structure

\section{Software Design of Rapid Monitoring System}

The system software design refers to the $\mu$ cos real-time operating system, which is mainly based on the task scheduling mechanism and can guarantee the concurrent execution of multiple tasks.

\subsection{Communication protocol design of the application layer}

The pros and cons of the protocol design directly determine the degree of intelligence of the system. In order to reflect the degree of intelligence of the system, a protocol design was performed on the application layer of the system to realize the transparent transmission of data packet packing and parsing[9-10]. The data transmission mode adopts the data frame mode, and the transmission sequence is a binary byte stream.

At the same time, the CRC16 checksum algorithm is used for data in data transmission so that the accuracy of the data transmission is correct. During data acquisition, 
the cloud sever sends protocol commands through the convergence node and the acquisition node. After the sensors respond, the data is returned to the acquisition node. The acquisition node packages the data and uploads it to the convergence node, and finally the convergence node upload the data to the cloud sever.

The system can realize three kinds of data model: timing acquisition, broadcast acquisition, and threshold wake-up acquisition. Once the monitoring network is established, a one-to-many mapping relationship can be established between the convergence node and the acquisition node. The acquisition node can set a unique device number and assign a unique device address to each acquisition node according to each SX1278 RF module. For example, when the convergence node sends a message with the device number $0 \times 55 \mathrm{AA}$, the acquisition node address is the device number and it needs to be immediately make a response.

\subsection{Software design of the acquisition node and the convergence node}

The acquisition node not only can collect the data of onsite sensors, but also can upload and receive data and commands through the LoRa network[11-13]. After the acquisition node receives the command sent by the convergence node, it first performs $\mathrm{CRCl} 6$ check on the data to ensure the accuracy of the data, and then performs corresponding operations according to the frame command in the packet. After the execution, the corresponding data information is uploaded to the convergence node, and the software of the acquisition node is executed. The workflow chart of software of the acquisition node is shown in Figure 4.

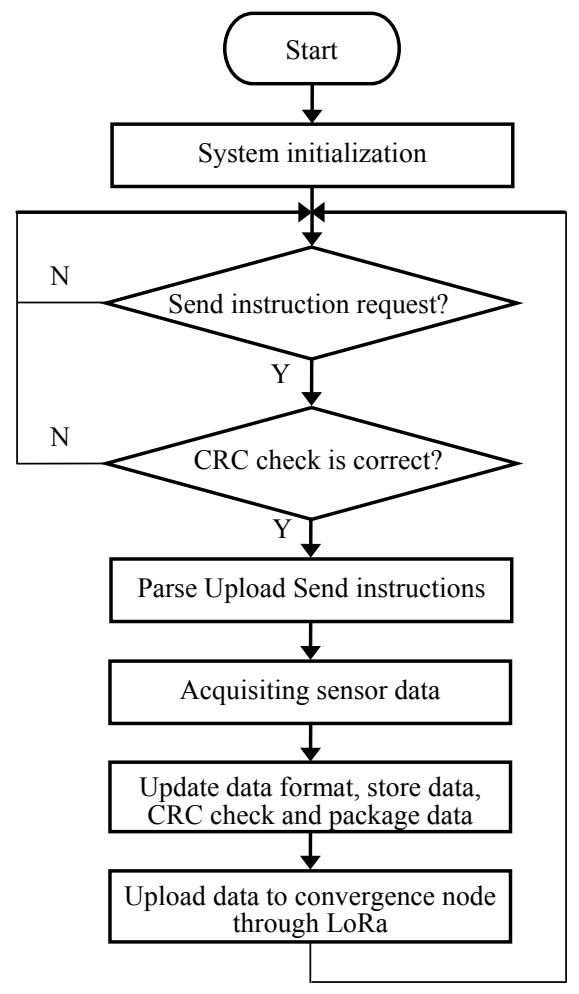

Fig. 4. The workflow chart of software of the acquisition node
The convergence node needs to communicate with the LoRa wireless network and also communicate with the GPRS public network. The flow chart of software design is shown in Figure 5. When the convergence node receives the data sent by the cloud server or the data uploaded by the acquisition node, it also performs a CRC check on the data to ensure the accuracy of the data[14$15]$.

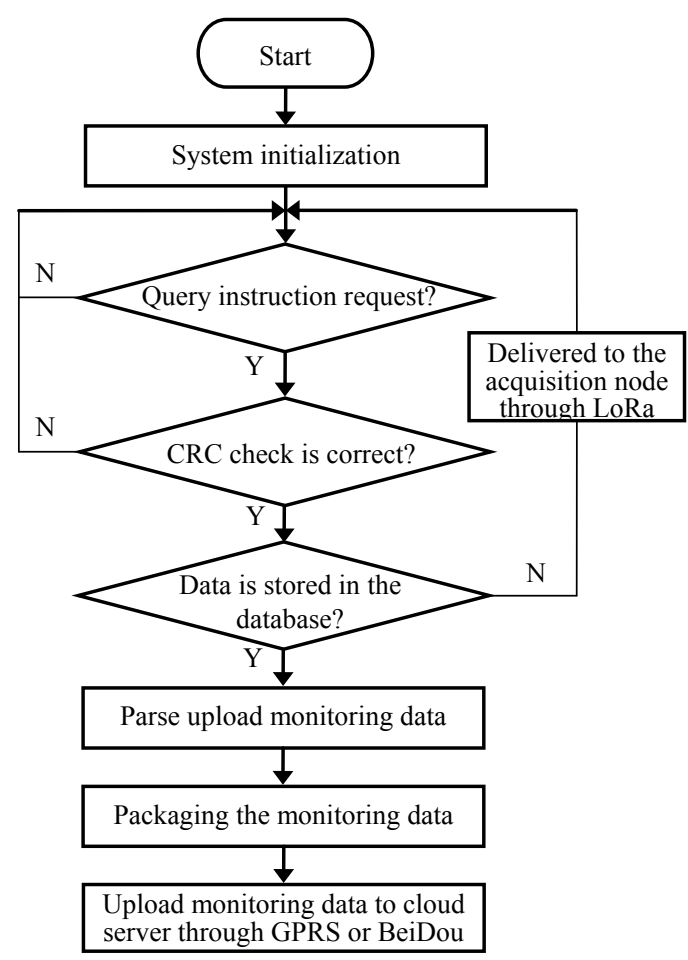

Fig. 5. The workflow chart of software of the convergence node

At the same time, the command in the packet determines whether the packet is sent by the cloud server or uploaded by the acquisition node. If the message is delivered, the device number is used to judge whether it is still operating on the acquisition node. If the message is uploaded and the CRC check of the received data is correct, it is uploaded to the geological disaster monitoring and warning cloud server through the GPRS network or the BeiDou satellite network. The workflow chart of software of the convergence node is shown in Figure 5.

\section{Conclusion}

This paper applies technologies of microelectronic, wireless communication and thin-film power technology to geological disaster monitoring system. This system uses low-power microprocessor, remote LoRa wireless network and BeiDou/GPRS public network to achieve real-time dynamic acquisition of geological disaster monitoring data. Specific data of geological disaster can be queried real-time in the remote data monitoring center or dedicated data receiving terminal, which provide data support for expert analysis and decision-making. Through the comparison and analysis, the new design of geological hazard monitoring system compared with the 
previous monitoring system has the following characteristics, such as shown in table 1.

Table 1. The Rapid monitoring system and Conventional system feature comparison table

\begin{tabular}{|l|c|c|}
\hline \multicolumn{1}{|c|}{$\begin{array}{c}\text { System } \\
\text { characteristics }\end{array}$} & $\begin{array}{c}\text { Rapid monitoring } \\
\text { system }\end{array}$ & $\begin{array}{c}\text { Conventional } \\
\text { sysytem }\end{array}$ \\
\hline Instrument weight & Light $\approx 4 \mathrm{Kg})$ & Heavy $(\approx 30 \mathrm{Kg})$ \\
\hline Instrument volume & small & large \\
\hline $\begin{array}{l}\text { Instrument } \\
\text { installation time }\end{array}$ & 1 hour & 4 hour \\
\hline $\begin{array}{l}\text { The instrument } \\
\text { response time }\end{array}$ & $30 \mathrm{~s}$ & $1 \mathrm{~min}$ \\
\hline $\begin{array}{l}\text { Instrument } \\
\text { transmission way }\end{array}$ & $2 \mathrm{G} / 3 \mathrm{G} / 4 \mathrm{G} / \mathrm{LoRa} / \mathrm{BeiDou}$ & $2 \mathrm{G} / 3 \mathrm{G}$ \\
\hline
\end{tabular}

The overall design of the system, hardware and software circuit design are introduced in detail. The system has the technical features of low power consumption, ad-hoc network, stable and reliable communication, and long transmission distance. The system completely meets the technical requirements for data collection of geological disaster and has a wide application prospect in the field of geological disaster monitoring.

\section{ACKNOWLEDGMENT}

This research was supported by the China Geological Survey project (DD20160283, DD20190639) and the National Key Research and Development Program of China (2018YFC150480502). We appreciate the help and support of Xiuding Cao, Yue Wu, Honglei Wang, Wei Guo of the Center for Hydrogeology and Environmental Geology Survey, China Geological Survey.

\section{References}

1. Hui-fu Zhang, Wei Kang, " Design of the Data Acquisition System Based on STM32", Procedia Computer Science, vol.17, 2013, pp.222-228.

2. Dan Chen, Zhixin Liu, Lizhe Wang, Minggang Dou, Jingying Chen, Hui Li, “ Natural Disaster Monitoring withWireless Sensor Networks: A Case Study of Data-intensive Applications upon LowCost Scalable Systems ", Mobile Networks and Applications, vol. 18, 2013, pp. 651-663.

3. Yonghua Song, Jin Lin, Ming Tang, Shufeng Dong, "An Internet of Energy Things Based on Wireless LPWAN", Engineering, vol 3, 2017, 460-466.

4. Mahmoud MS, Mohamad AAH, " A study of efficient power consumption wireless communication techniques/modules for Internet of
Things (IoT) applications ", Adv Internet Things, vol 6 2016, 19-29.

5. Joong-Soo Lim, " Design of High Speed Data Acquisition and Fusion System with STM32 Processor ", Journal of the Korea Convergence Society, vol. 7, 2016, pp. 9-15.

6. Jing Luo Cai, Guan Li, Xiang Li Bu, Xian Guo Liu, Hua Lu Xing, " The Design of Landslide Hazard Remote Monitoring System Based on STM32 MCU and GPRS ", Applied Mechanics and Materials, vol. 599-601, 2014, 1115-1119.

7. Dimitrios Loukatos, Ioannis Manolopoulos, Evangelia-Sofia Arvaniti, Kostas G. Arvanitis, Nick A. Sigrimis, "Experimental Testbed for Monitoring the Energy Requirements of LPWAN Equipped Sensor Nodes", IFAC-PapersOnLine, vol 51, 2018, 309-313.

8. Wang Chenhui, Wu Yue, Yang Kai. " Design of multi - channel data acquisition system based on STM32 ". Application of Electronic Technique, 2016, 42 (1) : 51-53, 57.

9. He Man-chao, " Real-time Remote Monitoring and Forecasting System for Geological Disasters of Landslides and ITS Engineering Application. Chinese Journal of Rock Mechanics and Engineering", 2009, 28(6): 1081-1090.

10. Yi-Bing Lin, Yun-Wei Lin, Chung-Yun Hsiao, Shie-Yuan Wang, " Location-based IoT applications on campus: The IoTtalk approach ", Pervasive and Mobile Computing, vol 40, 2017, 660-673.

11. Renato Macciotta, Michael Hendry, C. Derek Martin, “ Developing an early warning system for a very slow landslide based on displacement monitoring ", Natural Hazards, vol. 81, 2016, pp. 887-907.

12. Rashmi Sharan Sinha, Yiqiao Wei, Seung-Hoon Hwang, "A survey on LPWA technology: LoRa and NB-IoT", ICT Express, vol 3, 2017, 14-21

13. Lishan Baoa, Lei Weib, Chengling Jianga, Weiwei Miaoa, Bo Guoa, Wei Lia, Xiangdong Cheng, Rui Liu,Jun Zou, “ Coverage Analysis on NB-IoT and LoRa in Power Wireless Private Network" Procedia Computer Science, vol 131, 2018, 1032-1038.

14. W. Yang, M. Wang, J. Zhang, J. Zou, M. Hua, T. Xia and X. You, "Narrowband Wireless Access for Low-Power Massive Internet of Things:A Bandwidth Perspective," in IEEE Wireless Communications, vol. 24, 2017 , 138-145.

15. Matthias Jakob, I T. Owen, T. Simpson, "A regional real-time debris-flow warning system for the District of North Vancouver, Canada ", Landslides, vol. 9, 2012, 165-178. 